\title{
Camera fabula. La caméra, outil et objet sémiotique complexe en interaction
}

\section{Camera fabula. The video camera as a complex semiotic tool and semiotic object in interaction}

\author{
Julien Thiburce ${ }^{1 \text { a }}$ \\ ${ }^{1}$ Université Lumière Lyon 2, Laboratoire ICAR (UMR 5191)
}

\begin{abstract}
Résumé. L'enquête sur le sens des pratiques et par les pratiques en interaction se déploie aujourd'hui majoritairement par la mobilisation de la caméra. Les enregistrements audio et vidéo d'un événement offrent au chercheur la possibilité d'observer et d'attester de pratiques langagières et culturelles à différents niveaux de granularité (des traits syntaxiques et sémantiques aux aspects gestuels et corporels).

Néanmoins, son utilisation et son usage ne sont pas sans poser question, tant sur le terrain même de l'enquête que lors de sa programmation et de son traitement a posteriori. De la même manière que Umberto Eco (1979) a porté un regard sur le rôle du lecteur dans l'interprétation d'un récit littéraire, notre proposition questionne la place de la caméra dans l'expérience et l'interprétation de la ville en interaction, au cours d'une balade urbaine proposée par les musées Gadagne (Ville de Lyon). À la croisée de l'ethnométhodologie et de la sémiotique, la caméra émerge à l'intersection entre un outil qui permet d'attester d'une interaction et un objet qui participe de son déroulement par une perception instrumentée.
\end{abstract}

\begin{abstract}
Studies on the meaning of practices and through practices in interaction are nowadays predominantly driven by the mobilisation of movie cameras. Audio and video recordings of an interactional event give the researcher the opportunity to observe and document linguistic and cultural practices at different levels (from syntactic and semantic features to gestures and bodily aspects).

Nevertheless, its use and usage methodological and epistemological questions arise, in fieldwork as much as in video editing. Umberto Eco inquired into the role of the reader in the interpretation of literary narratives. Similarly, this work inquires the role of the movie camera in the display and the interpretation of an interaction in a city walk proposed by the musées Gadagne (Ville de Lyon). At the crossroads of ethnomethodology and semiotics, the camera emerges as a tool documenting interaction and an object participating in the unfolding interaction by instrumented perception.
\end{abstract}

\footnotetext{
${ }^{a}$ Adresse de correspondance : julienthiburce@gmail.com
} 


\section{Introduction}

Dans le champ des sciences du langage et des sciences de l'éducation, la caméra s'est massivement imposée en tant que ressource matérielle mobilisée pour l'observation et l'analyse des pratiques culturelles et langagières (Mondada, 2006). Par son utilisation, on dispose d'une trace et d'une conservation d'un événement situé et expérimenté depuis un certain point de vue que l'on peut jouer et rejouer en vue d'en saisir les différents niveaux de complexité - si tant est que le parcours de constitution des données n'a pas été semé d'embûches. Pour un regard allant de la syntaxe discursive au cours d'action des pratiques, les interactions sociales s'avèrent un terrain privilégié de la mobilisation de l'enregistrement audio-vidéo ${ }^{1}$.

Pour notre participation à la réflexion collective sur la notion de ressources, on appréhende le rôle de la caméra en tant qu'outil et objet complexe dans la pratique de terrain et dans l'interaction en sémiotique. Ainsi, une attention toute particulière est accordée à la manière dont elle participe de la formation d'un milieu spécifique (Ingold, 2013) au cours d'une d'interaction.

Dans le cadre de notre thèse (Thiburce, en cours), nous nous intéressons en effet aux discours et aux énonciations dans la ville en vue de saisir la tension qui se joue entre des usages tacites et conventionnels des espaces publics, d'un côté et des pratiques d'appropriation narrative et affective singulières, de l'autre. Une telle enquête souhaite ainsi accorder une importance majeure à la dimension écologique des pratiques langagières. En vue de procéder à une analyse située, nous avons constitué un corpus vidéo de visites guidées d'une durée de deux heures dans le cadre des Balades urbaines organisées par les musées Gadagne (musée d'Histoire de la Ville de Lyon). Nous avons enregistré deux réalisations d'un même itinéraire conçu par deux professionnels de l'image skateboardistique, itinéraire portant sur la pratique du skateboard à Lyon et les enjeux sociaux et politiques qu'elle sous-tend. Ce corpus a été constitué en s'appuyant sur une méthodologie développée au laboratoire ICAR en analyse des interactions, selon une perspective ethnométhodologique : deux caméras mobiles filment un même événement interactionnel depuis deux points de vue complémentaires et synchronisés.

Une première caméra enregistre les échanges multimodaux des interactants : c'est celle que manipule le chercheur en situation d'observation participante - ou filmante (Lallier, $2009)^{2}$. Pour ce qui est de la deuxième caméra, dans notre travail, elle n'est gérée ni par un chercheur ni par un ingénieur de recherche comme à l'accoutumée. Confiée aux participants eux-mêmes, nous avons fait l'hypothèse qu'elle favoriserait la saisie de

\footnotetext{
${ }^{1}$ D'un point de vue pratique, on peut proposer d'opérer une distinction entre audio-vidéo (production d'une piste audio et d'une piste vidéo séparées) et audiovisuel (production d'une piste audio et vidéo synchronisée).

${ }^{2}$ L'enquête des pratiques sociales par la caméra a été travaillé aux niveaux méthodologique et théorique dans une perspective sociolinguistique chez W. Labov (1972) avec son "paradoxe de l'observateur », en analyse conversationnelle chez H. Sacks (1984 : 21) pour les modalités d'enquêtes qu'elle favorise, chez P. ten Have (1999) dans la section de son manuel consacrée aux pratiques de constitution de données, chez S. A. Speer (2002) pour une problématisation des liens entre données «naturelles » et données « construites » ou « arrangées », chez M. Lynch (2002) pour une étude des liens entre collecte de données en milieu naturel et écologie des pratiques sociales, chez S. A. Speer \& I. Hutchby (2003) dans le cadre d'une réflexion sur les orientations et les conduites d'action des participants en rapport au dispositif de constitution de données. Dans une perspective sociologique, les travaux de C. Licoppe offrent un éclairage sur une gestion d'une diversité de médiations technologiques en interaction : du point de vue du chercheur comme depuis celui des acteurs sociaux, ces médiations donnent matière à enquêter sur les formes de la présence et leurs résonnances avec une pluralité d'acteurs sociaux (Licoppe, 2012).
} 
processus de sémiotisation de l'espace urbain qui émergeraient de leur propre enregistrement ${ }^{3}$. Ce procédé nous semble être une forme privilégiée d'attestation et d'accès à l'expérience sémiotique d'un environnement urbain en interaction.

Et s'il existe un environnement urbain, il est nécessaire d'appréhender sa transformation de manière dynamique, non pas en tant qu'entité sémiotique déjà-là et codée, qui se donne à percevoir et à savoir à des usagers de l'espace public, mais plutôt en tant que forme qui émerge de la négociation de soi à l'autre selon une interpénétration actorielle (Basso Fossali, 2012). À l'aune d'une telle réflexion se développe un regard sur la caméra comme ressource mobilisée par et pour le chercheur, afin de saisir un processus de sémiotisation de la ville en cours d'instauration. Ainsi, par l'analyse d'une collection de trois extraits qui se font suite dans l'interaction, nous verrons en quoi la caméra s'avère être une ressource au statut complexe, voire ambivalent, dans la co-gestion de l'expérience et de l'interprétation.

\section{Terrain de recherche et constitution de données}

Dans son Lector in Fabula, Umberto Eco (1979) a problématisé la place du lecteur dans l'interprétation de textes littéraires. Sa réflexion a notamment porté sur les différents régimes d'intentionnalité propres à l'auteur (intentio auctoris), au lecteur (intentio lectoris) et à l'œuvre littéraire produite (intentio operis) envers laquelle il convient de porter un certain « respect » (herméneutique et philologique). Ainsi, il s'est attelé à la systématisation des diverses «stratégies» énonciatives en jeu dans l'opération de sémiose $e^{4}$, tant en production (auteur) qu'en réception (lecteur) : comment l'auteur énonce-t-il un récit ? pour dire/faire quoi ? à qui ? et quelles positions le lecteur prend-t-il dans son parcours du texte ?

À l'instar d'Umberto Eco, par son regard porté sur la « coopération interprétative » du lecteur et de l'auteur dans l'émergence d'une expérience sémiosique, nous proposons ici une réflexion sur le rôle de la caméra dans l'interaction, de manière dialectique. On cherche en effet à questionner la place et le rôle de cette médiation technologique dans le tissage d'une expérience et d'une énonciation collective de la ville.

La caméra est un dispositif d'attestation d'une pratique sociale et de production d'un document dans et sur l'espace urbain ${ }^{5}$ : elle permet au chercheur l'enregistrement et l'archivage d'une situation d'interaction en vue d'en produire une analyse a posteriori. Son utilisation nécessite alors d'être programmée en amont du terrain, par rapport à une question de recherche propre à une étude. Dans le même temps, la caméra est un artefact aux positions actorielles et aux rôles actantiels indéterminés dans un récit co-construit par les interactants: si la projection d'une situation et la formulation d'hypothèses par le chercheur sur la mesure dans laquelle la caméra participerait d'une expérience in situ et in vivo sont nécessaires à la préparation de l'enquête, il serait en revanche malavisé de statuer a priori sur son usage et son utilisation dans le cours d'une expérience à venir.

Ainsi, il nous paraît pertinent de nous attarder sur les diverses stratégies mises en œuvre dans la pratique du terrain par le chercheur et sur la praxis située déployée par les participants, aux niveaux langagier et praxéologique. Aussi, parfois, nous ferons appel au hors-champ de l'enregistrement en mobilisant des informations nécessaires à la compréhension du contexte et de la situation d'interaction.

\footnotetext{
${ }^{3}$ Notamment par des procédés de cadrage (sélection d'un champ et d'un hors-champ), de focalisation (sélection et attention particulières portées à une portion de l'espace) de zoom (rapprochement ou éloignement d'une portion de l'espace).

${ }^{4}$ L'opération de sémiose consiste en l'articulation d'un plan de l'expression et d'un plan du contenu au niveau textuel ainsi qu'en l'articulation d'un signifiant et d'un signifié au niveau du signe.

${ }^{5}$ Cette dialectique entre un récit dans l'espace et sur l'espace peut être appréciée par une articulation entre un espace de l'énonciation (interactionnel) et un espace énoncé (en interaction), telle qu'elle a été formulée par A. J. Greimas Pour une sémiotique topologique (1976).
} 
Nous illustrons les principaux éléments qui permettent de situer l'interaction étudiée ici :

- $\quad$ au niveau spatio-temporel : elle s'est déroulée à Lyon, le dimanche 19 juin 2016, de $15 \mathrm{~h}$ à $17 \mathrm{~h}$;

- au niveau thématique : intitulée Esprit skate, cette balade urbaine a pour thème la pratique de la ville par les skateurs et les enjeux sociaux et politiques afférents dans la conception et l'aménagement de l'espace public ;

- $\quad$ au niveau actoriel : elle a été conçue et réalisée par deux photographes et vidéastes qui ont fait leurs débuts dans le milieu du skateboard et travaillent à un niveau international, avec des skateurs, dans le monde de la mode (Hermès) ou lors d'événements culturels (Nuits de Fourvière à Lyon); les participants sont des particuliers qui se sont inscrits à distance, par téléphone ou par internet, auprès d'un service dédié de la Ville de Lyon.

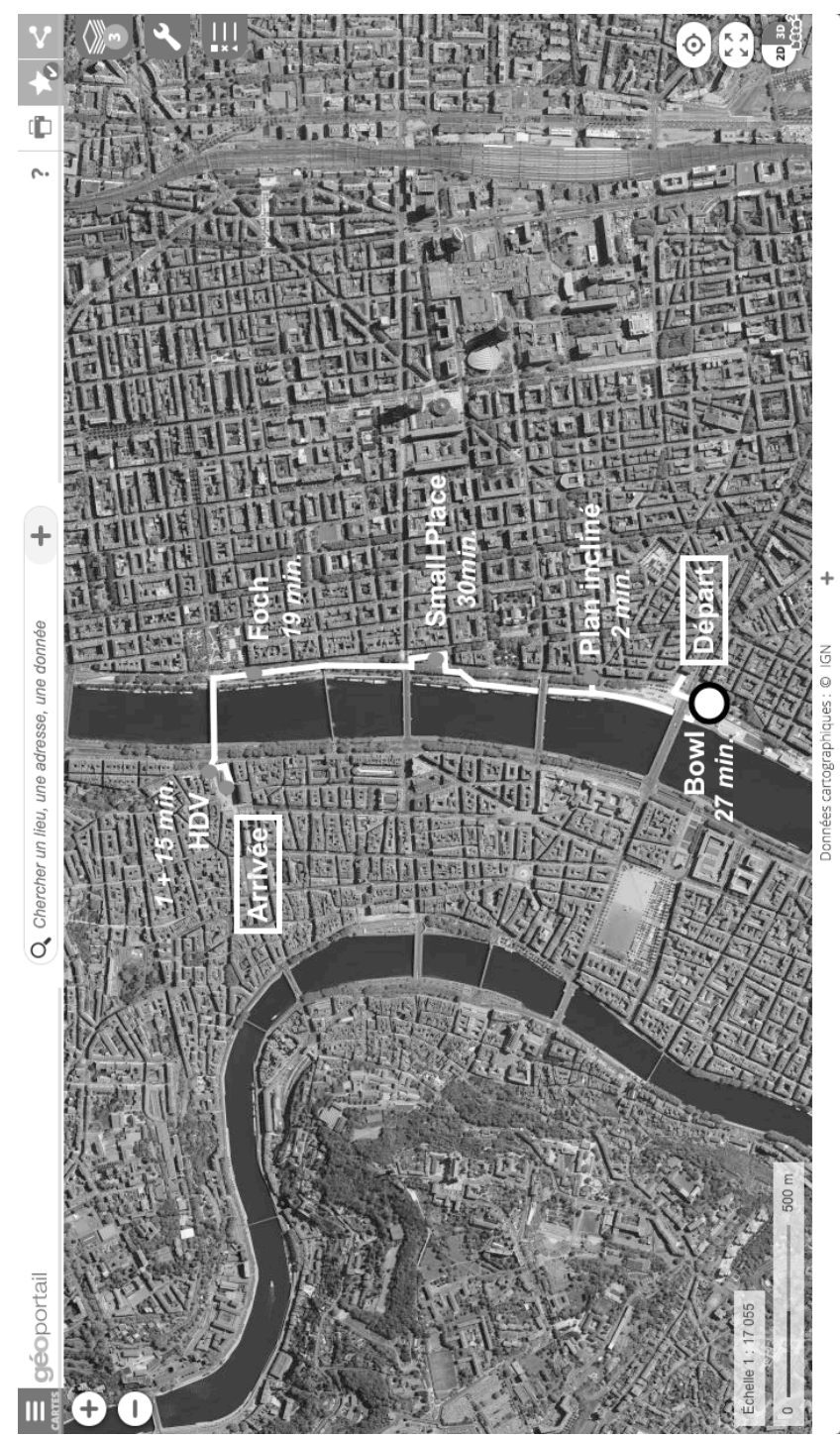

Figure 1. Parcours de la visite guidée Esprit skate - 19 juin 2016 
Ci-dessus, nous retraçons le parcours de la ville d'une ligne continue dont les points de départ et d'arrêts se situent respectivement dans le $3^{\text {ème }}$ et le $1^{\text {er }}$ arrondissements lyonnais. Les points d'arrêts sont instanciés par un cercle et par le nom que leur ont donné les guides (et d'autres skateurs). La durée de ces arrêts est indiquée à côté de chaque nom.

Les extraits de la collection constituée pour notre analyse sont ceux d'un échange au Skatepark de la Guillotière ( $7^{\text {ème }}$ arrondissement de Lyon), aussi appelé « le Bowl », marqué d'un point blanc ${ }^{6}$. Jour de match de l'Euro de football 2016 (UEFA), le parcours s'est déroulé dans une ville foulée par nombre de personnes et comptait sept participants initiaux : les deux guides, quatre personnes inscrites et nous-même. Au fil de la balade urbaine guidée, par des rencontres inopinées ou provoquées, des passants que les guides connaissaient se sont trouvés être participants à l'interaction.

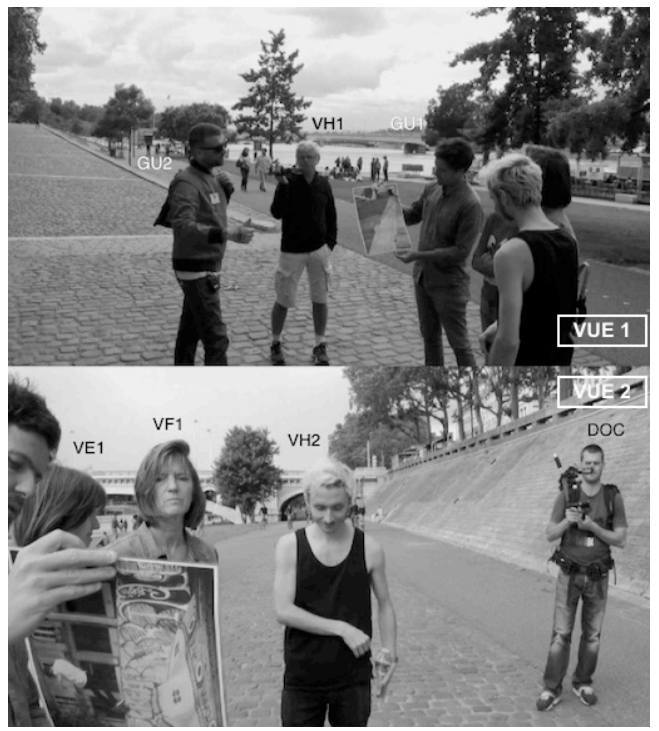

Figure 2. Dispositif d'enregistrement

La figure 2 ci-contre est une capture d'écran d'une vidéo des deux caméras synchronisées en post-traitement via le logiciel Final Cut ${ }^{\circledR}$. D'une part, elle permet l'identification des interactants par leur annotation et leur anonymisation? D'autre part, elle présente au lecteur le mode sur lequel l'enregistrement a été réalisé, ainsi que le dispositif conçu et mis en œuvre.

La VUE 1 est celle de l'enregistrement réalisé par nous-mêmes à l'aide d'un steadycam qui sert à rendre plus confortable la pratique d'enregistrement, à stabiliser l'image à analyser, à éviter la nausée à la visualisation.

La VUE 2 est celle de l'enregistrement réalisé par les participants avec la caméra que nous leur avons confiée tenue "au poing" (voir VH1 ci-contre).

Complémentaires, ces deux caméras font entrer en dialogue deux prises de vue de l'expérience en cours : une perspective étique, notre regard et notre posture par la caméra, et une perspective émique, avec la caméra dans le groupe (Pike, 1967) ${ }^{8}$. Les captures d'écran issues des extraits diffusés lors du colloque, accompagnées d'une transcription, donneront au lecteur un aperçu du film produit et analysé.

\footnotetext{
${ }^{6}$ Pour réaliser ces annotations, nous avons mobilisé une vue aérienne à l'échelle $1: 17055$ via le site www.géoportail.fr. Ce site offre aux utilisateurs un large choix de fonds de carte (vue aérienne, parcelles cadastrales, plan IGN, carte IGN, etc.) et une grande variété d'annotations à différents niveaux thématiques (agriculture, économie, culture et patrimoine, santé et social, etc.).

${ }^{7}$ GU1 signifie GUide 1, VH1 Visiteur Homme 1, VF1 signifie Visiteur Femme 1, VE1 Visiteur Enfant 1 et DOC DOCtorant. L'anonymisation est une étape du traitement de données parmi les annotations et transcriptions, à partir de conventions mobilisées en analyse des interactions par le Groupe ICOR : http://icar.univ-lyon2.fr/projets/corinte/documents/2013_Conv_ICOR_250313.pdf

${ }^{8}$ Néanmoins, la dichotomie n'est pas si nette que cela dans les faits : le chercheur (ici nous-même) est lui aussi renvoyé aux valorisations émiques de son propre domaine de travail, autant que de celles de l'espace social dans lequel son travail se déploie. Ainsi, en tant que chercheur, on peut porter un regard étique et un regard émique sur la scène en cours, et opérer des traductions de l'un à l'autre.
} 


\section{Faire par et avec la caméra : canaliser l'expérience ?}

La notion d'expérience est pour nous le tenant d'une réflexion sur les dimensions esthétique et éthique de la vie quotidienne. En travaillant sur des pratiques langagières situées, nous tentons d'appréhender l'émergence d'une diversité de rapports à l'espace urbain sur un gradient dont les extrêmes seraient, d'une part, des rapports tacites et normés et, d'autre part, des rapports intimes et singuliers : c'est là une tension entre le caractère hétérogène et le caractère ordinaire des pratiques sociales (Thibaud, 2002).

Si les interactants sont dans la ville en situation de balade urbaine guidée, d'autres personnes à l'entour font du roller, déjeunent ou attendent quelqu'un. Petit à petit, une scène émerge et un espace se tisse à partir de leur activité, en tension avec celles des autres. Pour la première séquence de notre collection - de $(00: 26: 14)$ à $(00: 27: 40)$-, nous nous demandons dans quelle mesure la caméra canalise l'expérience, terme ici mobilisé dans toute sa polysémie. En effet, canaliser l'expérience, ne serait-ce pas passer d'un échange in situ et in vivo à l'une des traces possibles par un canal audio-vidéo ? Canaliser l'expérience, ne serait-ce pas (faire) orienter et conduire un cours d'action particulier via un canal (phénoménologique et passionnel), allant rétrécissant ou s'élargissant au fil de l'échange ? À ce propos, attardons-nous maintenant sur la première séquence qui articule deux phases à travers lesquelles la caméra se révèle être entrave et adjuvant.

\subsection{La caméra, une entrave à l'activité de groupe ?}

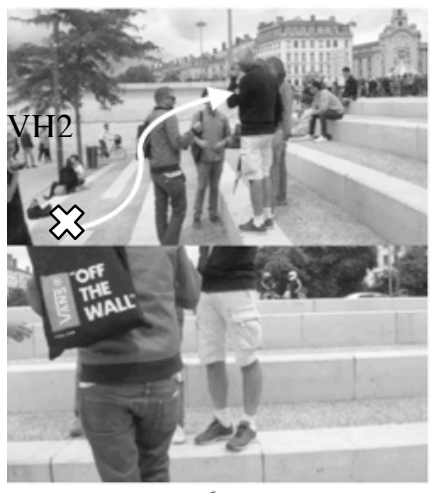

1

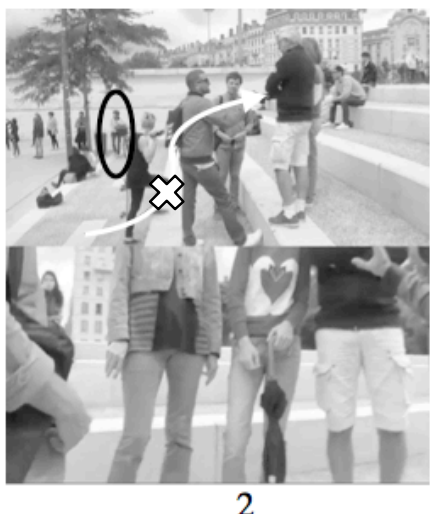

2

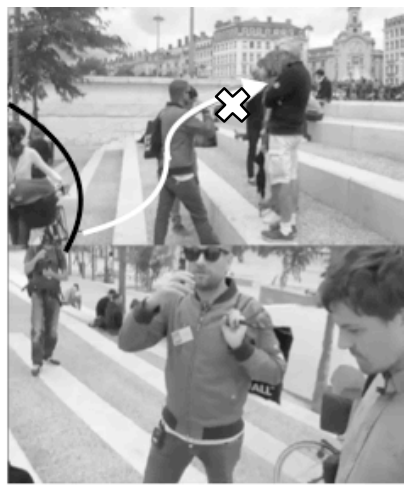

3

Figure 3. Pratique de la caméra, déplacement et distanciation

Dans la première phase, les guides développent une énonciation sur le rapport que les skateurs entretiennent à l'espace, une «façon d'appréhender la ville [...] complètement différente [...] de quelqu'un qui se déplace normalement » selon GU2.

Dans la figure 3, la caméra émerge comme une entrave à l'activité de groupe. En effet, la pratique de la caméra fait positionner $\mathrm{VH} 2$ d'une manière particulière par rapport au groupe : il reconfigure sa position par rapport aux interactants - y compris nous-mêmes - et par rapport à son entour. Sa trajectoire, marquée ci-dessus par une flèche blanche, se dessine dans un environnement où d'autres pratiques en cours participent de la transformation de l'espace. $\mathrm{VH} 2$ accomplit une multiplicité d'actions de manière simultanée : il filme, il marche, il observe la trajectoire et les pratiques des passants.

C'est là une forme de body torque (Schegloff, 1998; Pecqueux, 2014), une torsion de l'attention par des actions simultanées n'étant pas exécutées au même niveau de granularité ni au même plan. VH2, en dirigeant son attention vers la dame au vélo qui se trouve au loin et s'approche du groupe (fig. 3, images 2 et 3 , encerclée en noir), ne porte plus une grande 
attention à la manière dont il filme l'interaction en cours : ceci se remarque notamment par la déstabilisation du cadrage (fig. 3, vue 2 de l'image 2). Pour VH2, qui manipule la caméra depuis le début de la visite guidée, être pris dans le cours de l'enregistrement de l'interaction implique ici un désengagement de l'activité de groupe et une distance (au moins physique) dans le cours de l'interaction. Ce déplacement et ce recul se déploient dans un espace où des pratiques hétérogènes influent sur l'interaction en cours autant qu'elles peuvent être influencées par celle-ci.

\subsection{La caméra, un adjuvant de l'expérience esthétique ?}

Dans la deuxième phase, les guides proposent une description d'un élément précis : un « antiskate ». Inséré entre deux blocs de pierre, l'antiskate est conçu et mobilisé par les aménageurs de l'espace public pour empêcher la pratique du skateboard sur le mobilier urbain. Dans toute la multimodalité de l'interaction, divers mouvements sont opérés par la parole verbale et celle corporelle qui participent, de concert, à la caractérisation de la ville. Ici, un autre rapport à l'espace se déploie pour VH2 via la caméra, en relation à la description que GU1 et GU2 font de l'antiskate (encerclé en blanc ci-après).

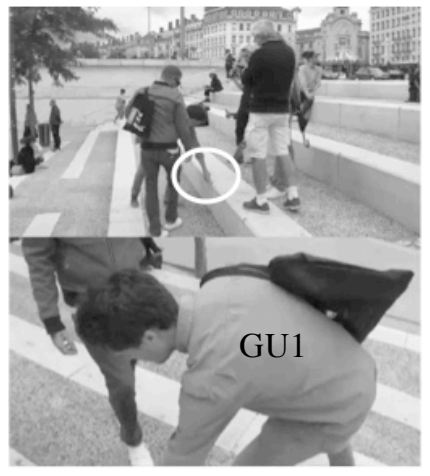

1

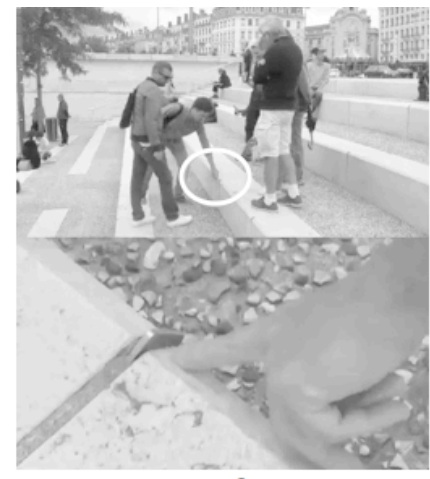

2

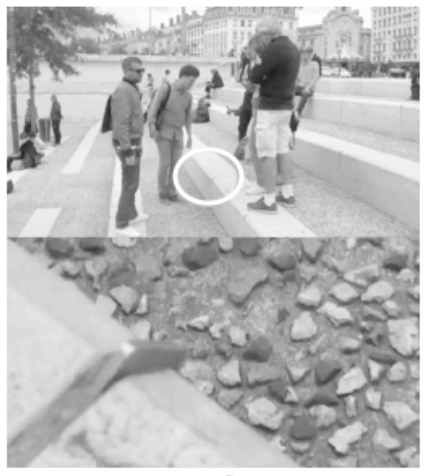

3

Figure 4. Focaliser l'attention et ajuster le regard

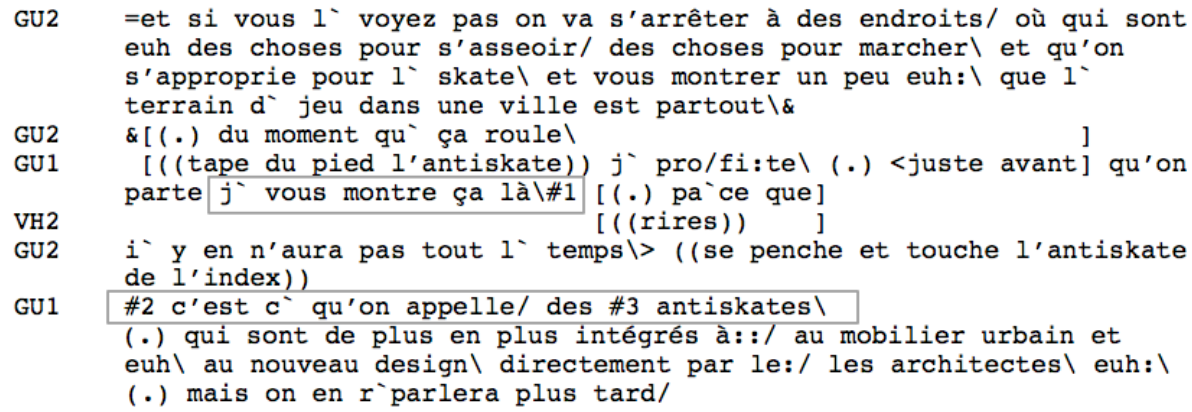

Transcription 1 - Antiskate

Si la caméra est une entrave à la participation d'un acteur à l'activité de groupe, elle se trouve être également un adjuvant dans l'expérience située de la ville. Par la manière dont VH2 manipule cette caméra, celle-ci émerge en tant qu'instrument favorisant un certain rapport phénoménologique aux objets de la ville et aux objets du discours. 
On observe cette « affordance » (Gibson, 1979) de l'objet caméra ${ }^{9}$ par les focalisations et les zooms qui peuvent être appréhendés depuis les liens entre les modalités visuelle, corporelle et verbale. En effet, GU2 propose de focaliser l'attention collective sur un antiskate (lignes 26-28) ${ }^{10}$ pour le développement d'une énonciation sur un esprit skate ${ }^{11}$. Dans la négociation d'une énonciation et d'une perception de cet objet convoqué, VH2 opère un mouvement de son corps et de la caméra vers l'antiskate en question.

Ce mouvement peut se découper en trois phases: une première phase est celle du recadrage du champ de l'énonciation et de la perception des interactants vers l'antiskate (\#1) ; une deuxième est celle du zoom sur l'objet en question (\#2); une troisième phase est celle de l'ajustement et de l'accommodation de la perception, en vue d'opérer une focalisation sur l'antiskate pour un champ net et une bonne résolution (\#3). Une quatrième phase pourrait être ajoutée : le recadrage de l'attention vers les interactants.

Ainsi, pour une réflexion sur l'expérience esthétique de l'espace urbain et les postures éthiques de chacun, l'attestation de re-cadrages aux niveaux narratif et perceptif donne des indices sur la mesure dans laquelle une ré-entrée se déploie entre le langage et la perception. Le discours des guides dans la ville (espace de l'énonciation) et sur la ville (espace énoncé) influe sur la perception qu'ont les participants de leur entour et, par là même, les objets de la ville émergent non seulement à travers la description qui en est faite, mais aussi par une focalisation attentionnelle réalisée de manière multimodale (gestes, postures, regards) et de manière (re)médiée (recadrages, zooms, accomodations). Que ce soit de manière instrumentée (par la caméra) ou non (par le corps), les interactants tissent leur interaction par une prise (Landowski, 2009) aux objets alentour qui évolue au fil du parcours. Entre un détachement du corps et un rapport de proximité maximale à l'objet, une relation presque osmotique, les participants appréhendent l'espace de l'énonciation et développent l'espace énoncé de manière plastique. Par la mobilisation et la co-gestion de ressources multimodales et praxéologiques, les interactants co-construisent et négocient la matérialité même de l'espace.

\subsection{Une ambivalence de la caméra sur le terrain}

Ainsi, entre entrave et adjuvant de l'expérience in vivo, la caméra pour et par le chercheur émerge comme un objet ambivalent sur le terrain. La caméra émerge comme un objet ambivalent dans la négociation de la scène pratique, entre observation (évaluative) et participation (immersive) qui implique une co-gestion de l'activité par le chercheur.

D'un côté, cette médiation technologique est un outil pour servir une étude de recherche en sciences du langage sur l'expérience esthétique et l'appropriation narrative de la ville: elle favorise une analyse et une description a posteriori de l'interaction située. De l'autre, la caméra est un objet dont les caractéristiques se manifestent par (i) une pratique déjà réalisée

\footnotetext{
${ }^{9}$ Et en cela notre propos s'inscrit en continuité de la réflexion proposée par E. De Stefani au colloque (Les objets matériels dans l'interaction sociale : ressource disponible ou constituée ?). L'objet est moins appréhendé dans une matérialité qui se «donne » à voir et à manipuler qu'en tant que forme «constituée » par l'interaction, selon les niveaux et critères de pertinence négociés par les pratiques des acteurs sociaux.

${ }^{10}$ Au niveau syntaxique, on note un aspect tout à fait intéressant dans la mobilisation du pluriel par GU1. Alors qu'il pointe et touche un antiskate particulier, il dit qu'il s'agit de ce «qu'on appelle des antiskates »: la description de cet antiskate vaut pour d'autres antiskates (contigus) ou non (in absentia).

${ }^{11}$ En reprenant des catégories développée en linguistique de l'énonciation, on voit bien que cette description participe d'une énonciation plus large, en relation à une argumentation et à une narration dans la co-construction d'un récit social par lequel émergent des tensions des pratiques de l'espace public.
} 
et introjectée (processus de cadrage, de sélection d'un champ et de gestion de la profondeur par accommodation) et (ii) une pratique créative dans une configuration spécifique (qui modifie la configuration en place). Entre ces deux pôles que sont l'introjection et la créativité, la caméra est en tension entre une caméra-type (définition encyclopédique et pratique ubiquitaire) et une caméra-occurrence (champ de pertinence spécifique de la pratique en relation à un cours d'action).

La caméra permet la constitution de données sur une expérience in situ et in vivo en vue d'opérer une thick description (Geertz, 1973), une caractérisation dense et fine d'une interaction locale pour une réflexion sur les pratiques culturelles. À partir de cette thick description, on pourrait s'interroger sur la possibilité d'une généralisation de la scène locale par le repérage, le marquage et l'annotation de variants et d'invariants structurels. Autrement dit, à partir d'une mise en perspective de la pratique de la caméra au cours de balades urbaines guidées (et dans d'autres situations), on systématiserait les modalités et les modalisations de présence en jeu dans la gestion de l'interprétation par la manipulation de la caméra en interaction.

Au-delà de notre recherche, la caméra implique un positionnement dans trois phases du travail de terrain :

(i) en amont de l'interaction in situ et in vivo, en se demandant pourquoi et comment mobiliser cette caméra, en déterminant les instructions ou consignes à (se) donner ;

(ii) dans le cours de l'interaction, en observant l'ici et maintenant qui se tisse dans la relation aux participants et à l'environnement interactionnel ;

(iii) en aval, en mesurant l'écart entre la projection de l'utilisation à venir du dispositif et la pratique en acte et réalisée, non seulement afin d'évaluer le caractère approprié de ce dispositif, mais aussi de prendre la mesure des modifications à opérer sur ce dispositif pour des utilisations futures dans des configurations semblables (ou non).

Par là même, depuis le point de vue du chercheur, la caméra est un artefact expérimental et technique qui permet d'archiver et de documentaliser l'expérience, en vue d'en faire une analyse a posteriori. Afin de questionner la pertinence et de motiver l'utilisation d'une caméra dans une recherche sur la socialisation de l'expérience et sur l'expérience socialisée, la pratique déployée par le chercheur doit ainsi être évaluée à chacune de ces trois phases. Aussi convient-il d'enquêter sur les modalités par lesquelles cette médiation devient ressource dans l'expérience située, parmi d'autres ressources potentielles. Catalyseur, adjuvant ou entrave à l'interaction en cours pour les participants à qui nous avons proposé de l'utiliser, la caméra joue un rôle qui n'est connu qu'en partie: par une partie en cours, qui rejoue localement la place de cet outil de la recherche, et de manière partielle, parce que toujours en relation avec un acteur social à rencontrer ${ }^{12}$.

Dès lors quels faire se déploient dans l'expérience de la ville en interaction par la manipulation de cette caméra? Jusqu'à quel point la caméra participe-t-elle de la modalisation des acteurs sociaux comme celles de l'espace urbain?

\section{La ville et ses (por)traits, entre -notation et -scription}

En traçant et en suivant cette piste de réflexion sur la dimension située de l'expérience sémiosique, une analyse sémiotique de l'interaction permettrait d'étudier la manière dont la caméra participe de la co-construction d'un récit social. Maintenant, nous allons essayer de voir quels sont les (por)traits qui émergent pour les acteurs sociaux (coprésents ou non), pour l'espace et pour la caméra dans la scénarisation du récit en interaction (spatialisation,

\footnotetext{
${ }^{12}$ En cela même, il nous semble que la pratique de terrain ne peut passer que par une «stéréoscopie » non-stéréotypique dans l'articulation des valorisations étiques (du chercheur) et émiques (des acteurs sociaux).
} 
temporalisation, actorialisation, modalisation). Dans la deuxième séquence de notre collection ((de 00:27:40 à 00:28:27)), à la suite de la description de l'antiskate, VH2 propose à VF1 de lui passer la caméra en se dirigeant vers le point deuxième point d'arrêt. Il opère une focalisation sur cette dernière en la thématisant et en la caractérisant d' «engin $»^{13}$. Dans notre analyse, on se concentre sur les ressources mobilisées par les interactants dans l'élaboration d'un faire sémiosique, entre -notation et -scription.

\subsection{Passer et prendre le relais : agentivité et passivité}

Cette thématisation de la part de $\mathrm{VH} 2$ est tout à fait cruciale dans la structuration de l'échange et la reconfiguration des positions actorielles. Ce passage de relais dans la pratique de la caméra de VH2 vers VF1 implique une forme de négociation de :

(i) ce que l'on peut faire de cette caméra et avec cette caméra dans la balade urbaine : si VH2 l'a utilisé d'une certaine manière, quelle était cette manière ? VF1 doit/peut/saitelle faire de même ? comment se tenir vis-à-vis des co-participants avec la caméra et comment la tenir?

(ii) la gestion de l'espace de la balade urbaine avec cette caméra : comment gérer son rapport à l'entour et aux autres interactants ? peut/doit/sait-on gérer une interpellation et une sollicitation sur plusieurs terrains de manière simultanée ? dans quelle mesure ce passage implique-t-il une gestion de la transformation de sa position actorielle dans le parcours éphémère de la ville?

Même si très succincte, la transcription ci-après illustre bien l'hétérogénéité constitutive de l'expérience, les asymétries entre les performances des participants.

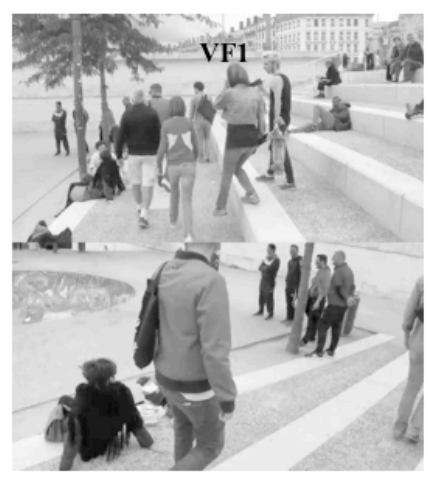

1

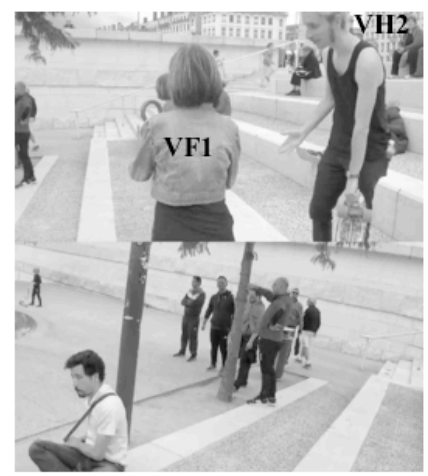

2

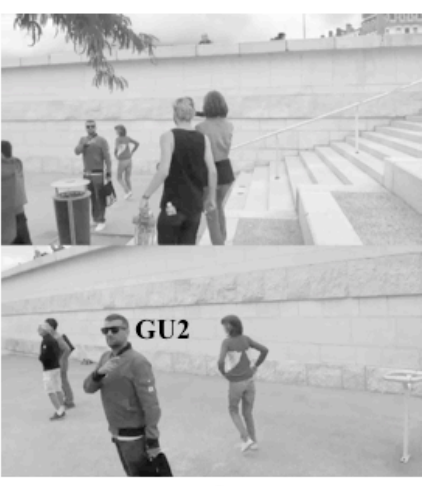

3

Figure 5. Passer et prendre le relais

\footnotetext{
${ }^{13}$ Pour lui, l'espace pratiqué avec la caméra serait-il ainsi un chantier où mettre de l'ordre plus qu'un terrain déjà praticable de manière confortable ?
} 


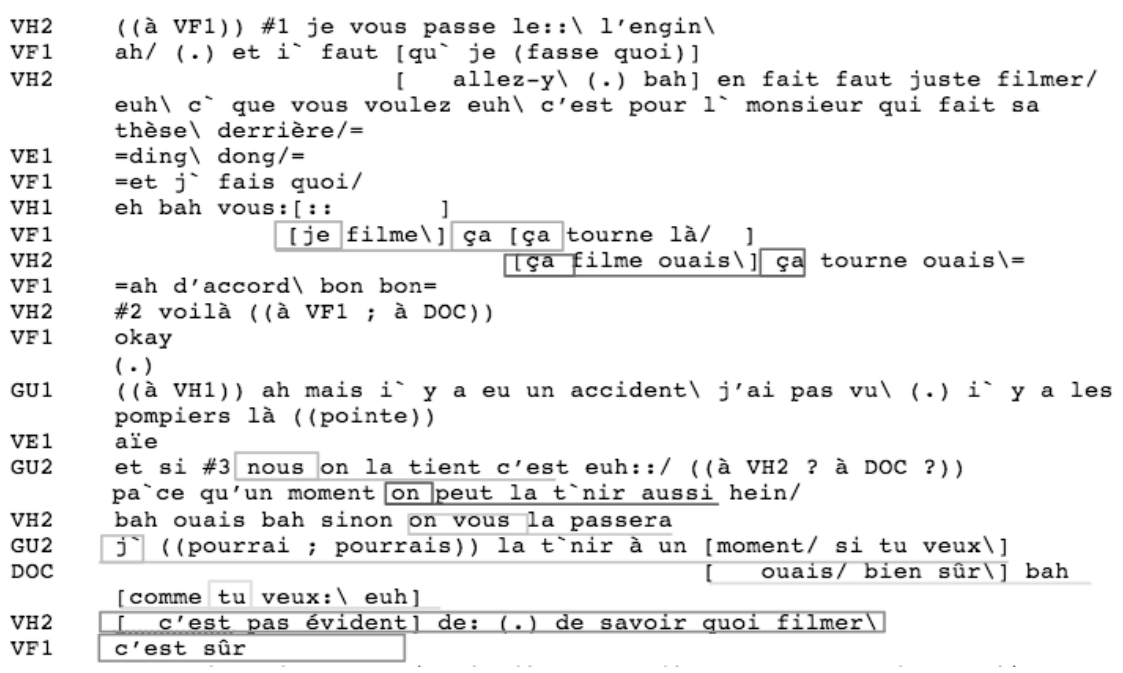

Transcription 2 - Engin

Au niveau sémiosique, interprétatif et praxéologique, le cours d'action de VF1 se trouve déstabilisé par la proposition de VH2, entre falloir et vouloir. D'une part, on voit que VF1 ne sait que faire de cette caméra proposée par $\mathrm{VH} 2$ et (se) demande à quel programme d'action elle doit s'adapter (ligne 8) : «i faut qu' j' fasse quoi »? D'autre part, on se questionne sur le degré d'agentivité dans la conduite et la tenue de la caméra par VH2 : «allez-y, bah en fait faut juste filmer ce que vous voulez» (lignes 9-10). Pour les participants, la caméra créé une tension dans l'acte filmique ${ }^{14}$ entre manipulation immédiate et action programmée. Cette tension instancie une possible discontinuité entre deux référentiels : le référentiel des performances introjectées et projetées par les participants et le référentiel des attentes du chercheur telles que les interactants peuvent se les projeter.

D'un côté, il y a en effet une immédiateté pour les participants - voire un automatisme et une passivité : on repère cette passivité chez VF1 et VH2 dans un énoncé comme "ça tourne » (ligne 15) ou «ça filme» (ligne 16) et chez GU2, lorsqu'il dit « on la tient» ou « on peut la tenir » en pointant la caméra (lignes 24 et 26, image 3). Est-il alors possible de seulement tenir la caméra et de la laisser tout faire toute seule ? Sommes-nous dans une configuration où nous avons une passivité pleine ou bien une agentivité implicitée ? Si ces questions instancient les limites de notre interprétation, il est intéressant d'observer les différents degrés d'implication de GU2 par le passage d'un «nous » (ligne 24) à un «on » (ligne 25) pour aboutir à un « je » (ligne 27) : l'agentivité des participants, l'activation d'un rapport singulier et subjectif à la caméra se fait de manière progressive et scalaire.

D'un autre côté, on voit émerger une position dans laquelle les participants se trouvent par rapport à eux-mêmes et par rapport au chercheur. Lorsque VH2 dit que «c'est pas évident de savoir quoi filmer» (ligne 30), on se demande si cela n'est pas évident parce qu'il se passe trop de choses à observer et à faire entrer dans le champ de la caméra ou si cela n'est pas évident par rapport à l'objet de notre recherche et à notre intention, ou les deux.

Il nous apparaît ainsi que, dans le discours de la recherche en sciences humaines et sociales, lorsqu'on mobilise des expressions comme «faire du terrain » et «aller sur le terrain », la caméra est un objet qui met le chercheur dans une situation intermédiaire, entre stratégies et tactiques (de Certeau, 1980). En effet, on doit faire et conduire soi-même le cours de la constitution de données et, dans le même temps, on doit faire faire quelque

\footnotetext{
${ }^{14}$ En creux, on fait référence ici à L'acte photographique de Philippe Dubois (1983).
} 
chose à quelqu'un pour une recherche et un champ spécifiques : mais, dans une perspective de l'apprentissage et du développement de la recherche, quelles sont les "bonnes conduites" d'action? En l'occurrence, comment faire filmer des personnes en situation de balade urbaine? Jusqu'où accompagner les participants dans l'utilisation d'une médiation importée par nous-même sans influencer son usage (normé) et sa pratique (située) ?

Ces questions mettent en jeu une transitivité du chercheur vers les participants, mais aussi, dans un autre mouvement transitif, des interactants vers le chercheur: in situ, comment ce dernier peut-il ou doit-il répondre à des sollicitations et à des requêtes des participants ? Cette question entre en résonnance avec le moment où GU2 propose de tenir la caméra dans l'extrait ci-dessus (lignes 24-25 et 27). Pour nous, la question s'est posée de savoir comment nous devions nous ajuster à lui sur son propre terrain de pratique de la balade urbaine, à son geste (celui d'aider VF1 et VH2 ? d'utiliser la caméra pour luimême ?), à son intention et à son intentionnalité (Eco, 1979).

\subsection{La caméra-stylo : prendre et (d)écrire les données}

Décisif, ce passage de la caméra d'un acteur à un autre est en lien avec la gestion des tours de parole dans l'élaboration d'une énonciation de groupe. $\mathrm{Si}$, dans une pratique artistique la caméra est au vidéaste ce que le stylo est à l'écrivain (Astruc, 1992), comment est-elle tenue, manipulée et dirigée dans un travail en sciences humaines et sociales sur et dans une expérience de la ville en parcours ? Si l'on pense à la prise de données et à l'esthésie énonciative de Pierre Ouellet $(2000)^{15}$, qu'est-ce qui est perçu et (ré)énoncé par la caméra? selon quelles modalités et à quelles fins?

Pour tenter de répondre à ces questions, on peut considérer la présence du chercheur et sa pratique d'enregistrement de l'ici et maintenant selon deux modalités.

La première modalité est celle de la documentalisation d'une interaction dans l'espace urbain pour un après. Sur son versant document, la pratique d'enregistrement permet de revoir à nouveaux frais la notion de -notation dans le rapport aux données ${ }^{16}$, entre une perspective «étique » et une perspective "émique» (Pike, 1967). L'an-notation consiste en la production de données depuis de point de vue du chercheur sur et dans l'interaction (étique). La co-notation caractériserait une notation négociée et co-construite (KerbratOrecchioni, 2005) sur et par l'interaction, selon un processus de sémiose collaborative : les positions d'acteurs et les rôles d'actants de l'espace public émergent ainsi par l'opération de sémiose co-élaborée entre les participants. La dé-notation renverrait quant à elle à la suppression d'une notation produite par soi-même ou par autrui.

15 Pierre Ouellet (2000: 35-58), dans une perspective sémiotique sur l'interprétation des textes littéraires, propose un principe qu'il travaille dans le cours du chapitre intitulé «L'œil parlant. Poièsis perceptive et aisthèsis énonciative » : « la praxis énonçante est une ethèsis énoncée - qui en est moins le résultat que la condition, inscrite au sein même de la manifestation discursive ».

${ }^{16} \mathrm{La}$ notion et le processus de notation ont notamment été problématisés dans le cadre d'une sémiotique de l'action par Maria Giulia Dondero (2014), en regard à la notion et au processus de textualisation dans le déploiement d'une pratique. En cherchant à repousser les limites d'une analyse sémiotique des pratiques sans «trahir » les principes qui en assurent la consistance et le tenue, la sémioticienne a ainsi consacré un travail pratique et théorique sur la diversité des pratiques sémiotiques développant ces deux macro-catégories que sont la «textualisation » de l'action où en produisant un matériau in vivo et la "notation» ex post de l'action, à partir de matériaux déjà constitués. Pour notre part, il nous semble qu'une telle typologie situe ces deux modalités pratiques sur un pied d'inégalité. En suivant la proposition de François Rastier (2011), le texte serait à mettre en regard avec le document. La -notation ainsi peut descendre à un niveau inférieur de la typologie et caractériser différentes modalités de documentalisation. 
La deuxième modalité d'enregistrement d'un ici et maintenant dans un ici et maintenant est celle d'une textualisation de l'espace, aux niveaux esthétique, épistémique et passionnel. Sur son versant textuel, la pratique de la caméra permet de saisir une -scription multimodale et polysémiotique des valeurs et des valences entre des sujets et des objets dans la ville, d'une sémiotique (multimodale) à une autre (praxique). D'une part, nous avons une dé-scription d'un pli énonciatif d'une altérité : la pratique de la caméra favorise le palimpseste actoriel et participe de l'écrasement culturel dans la gestion de la "mémoire vive" d'une culture. D'autre part, la pratique de la caméra met en jeu une in-scription dans la ville par son propre pli énonciatif, à la fois individuel et collaboratif : par une mise à jour de la perception et de l'énonciation, les acteurs sociaux peuvent réinscrire et réévaluer leur présence dans un récit social toujours à négocier dans une archéologie des pratiques.

Pour le chercheur, il se déploie ainsi une «stéréoscopie » (Pike, 1967 ; Basso Fossali, 2015) pour l'observation et l'interprétation de l'expérience des acteurs sociaux. Quand bien même nous sommes en position d'observation participante, nos modalités de participation par la caméra ne sont pas du même ordre que celles des participants : notre pratique in situ est différente de la leur et, de facto, nous n'expérimentons ni ne remédions la même scène. Si nous sommes ici en train d'accomplir une activité située pour un après (l'analyse), un tel fait ne semble pas être premier pour les interactants (sauf dans le cas où la balade urbaine serait conçue de prime abord en tant que capitalisation de l'expérience).

\subsection{La situation, son enregistrement et son analyse : traduire l'expérience}

Ce dernier point nous permet alors de monter encore un peu plus en abstraction afin de questionner le faire du chercheur par rapport au « respect» de la pratique narrative cher à Umberto Eco. De la prise des données à leur traitement, les enregistrements impliquent une forme de traduction a posteriori en vue de rendre intelligible une séquence d'actions au niveau actoriel, spatial et temporel. Aussi, cette traduction n'est pas sans poser question quant à la préservation de l'agentivité et l'intentionnalité des (re)prises de parole, des gestes énonciatifs comme des silences. Il nous semble que décrire des données, narrer son analyse et défendre une argumentation, c'est proposer un point de vue sur une scène à appréhender avec prudence et précaution.

En effet, si la caméra et l'enregistrement favorisent l'interprétation d'une expérience in situ et in vivo pour le chercheur, notamment par la possibilité de jouer et de rejouer une séquence, le sens de l'expérience en train de se (trans)former débordera toujours du cadre et du champ de l'activité attestée. Le sens des pratiques langagières, par des expériences individuelles et de groupe, trouve une coloration, une texture et une profondeur qui ne peuvent être contenues totalement et entièrement là, dans l'enregistrement qui en est fait. L'enregistrement peut bien laisser des traces et attester d'une expérience en cours, mais le film n'est pas l'expérience elle-même : c'est là une tension entre la dimension figurative (encadrée) et celle figurale en jeu dans les processus sémiosiques ${ }^{17}$.

Afin de réduire l'indétermination de l'interprétation de l'enregistrement vidéo, les étapes qui se déploient dans la séquentialité de l'échange interactionnel sont repérées et caractérisées : c'est l'émergence, à partir des données, des cours d'actions des interactants et de leur «intelligibilité publiquement établie et reconnue» (Mondada, 2012: 130) accountability (Garfinkel, 1967) - que l'on interprète, en retour, par une approche située des pratiques langagières.

${ }^{17}$ La dimension figurative est celle encadrée et déployée par un dispositif topologique (Greimas, 1984) et la dimension figurale est celle qui fuit à travers l'expérience - voire qui peut constituer une échappatoire dans l'analyse, où ce sur quoi l'on ne peut avoir prise relèverait du figural. 
Et si cette intelligibilité émerge à partir des données déjà prises et à (re)traiter, elle nécessite surtout d'être perçue et saisie dans le cours même de l'enregistrement. La prise de données passe par la recherche d'une prise et d'une emprise sur le terrain en cours de constitution. Une tension s'y déploie alors entre les projections et les attentes du chercheur et les variations ou indéterminations du terrain de la recherche.

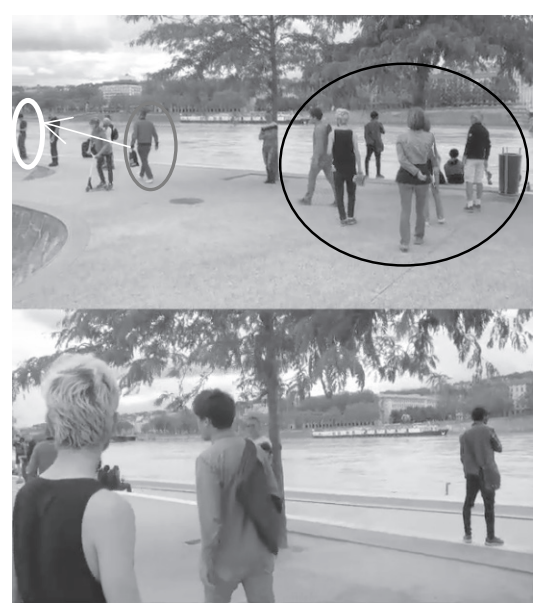

Figure 6. Scission du cadre et reconfiguration du champ

Dans notre cas, cette indétermination trouve notamment son ancrage dans des facteurs sociopragmatiques, par exemple lorsque GU2 (figure 6, cercle gris) va échanger avec une connaissance, « la babouche » (dorénavant BAB, dans le cercle blanc). En arrivant au skatepark de la Guillotière son skateboard dans une main et une bouteille de Volvic dans l'autre, ce dernier était venu saluer les guides plus tôt dans la balade par une rencontre fortuite.

À ce moment, le cadre de l'interaction se scinde en deux scènes et deux cadres distincts (Goffman, 1973) : le groupe attend (figure 6, cercle noir) que GU2 (figure 6, cercle gris) aille saluer BAB (cercle blanc) et revienne vers eux avant de poursuivre leur parcours de la ville. Dans le cours de l'enregistrement, nous nous sommes demandé que faire avec et par cette caméra. Quelle distance spatiale maintenir par rapport au groupe ? Où regarder et quel recadrage créer ? Devionsnous rester en retrait et garder les deux scènes à équidistance ou devions-nous suivre le groupe qui attendait GU2 ? Aussi, les questionnements se sont-ils poursuivi dans l'analyse : quelle focalisation de l'interaction peut-on opérer en post-traitement ?

Dans la partie suivante, on s'intéresse donc aux décisions prises par nous-mêmes et à leurs conséquences, non seulement sur la reconfiguration de l'espace interactionnel, mais aussi sur notre position sur le terrain par le discours des interactants.

\section{Prendre position et mettre en position : questions spatiales et éthiques}

Notre analyse s'oriente ainsi vers les effets (perlocutoires) de notre intervention par et avec la caméra sur le terrain. Cette intervention implique de prendre position soi-même par rapport à un groupe et de faire tenir une position aux acteurs observés dans un champ. Cette position est à apprécier tant dans une acception spatio-temporelle que dans une acception éthique dans le récit d'une expérience de la ville.

Lorsque nous prenons une place par rapport aux participants dans l'espace et le temps de l'interaction, nous faisons tenir une place particulière à ces derniers dans l'enregistrement réalisé par le cadrage opéré sur la scène. Dans le même temps, en étant en co-présence et en entrant en interaction avec les participants par un dispositif d'enregistrement, c'est non seulement tenir un rôle vis-à-vis d'eux in situ et in vivo, mais c'est aussi mettre en position ces interactants par une pratique dans et sur l'espace public.

Les prises de position de chacun influencent la scène et sont influencées par l'interaction en cours. Cette perméabilité et cette influence entre l'interaction et la scène pratique et celles entre l'entour et les conduites des acteurs peuvent être appréhendées par l'analyse de l'échange entre $\mathrm{BAB}$ et GU2, dans la troisième séquence de notre collection (ci-dessous). 
Dans le cours de l'enregistrement, nous avons pris la décision de mettre à équidistance les deux scènes et les deux cadres afin d'avoir une appréhension de la scène globale. En figure 7, nous proposons un zoom opéré en post-traitement sur GU2 (à droite) et BAB (à gauche), afin de mieux apprécier leur interaction au niveau multimodal. On s'attarde notamment à leurs énoncés sur le dispositif vidéo manipulé par nous-même et par VH2, au moment où $\mathrm{BAB}$ était venu saluer les guides. Et si jusqu'ici, ce qui a été principalement évoqué, c'est la place de la caméra dans le dispositif d'enregistrement, il est important de noter que le son y joue une place primordiale. Les guides étant équipés d'un micro-HF, nous disposons de deux pistes sonores distinctes pour un même espace visuel, de manière simultanée. Sans cela, nous n'aurions pu avoir accès aux mots échangés qu'en nous rapprochant d'eux (physiquement) et la scène analysée serait toute autre.

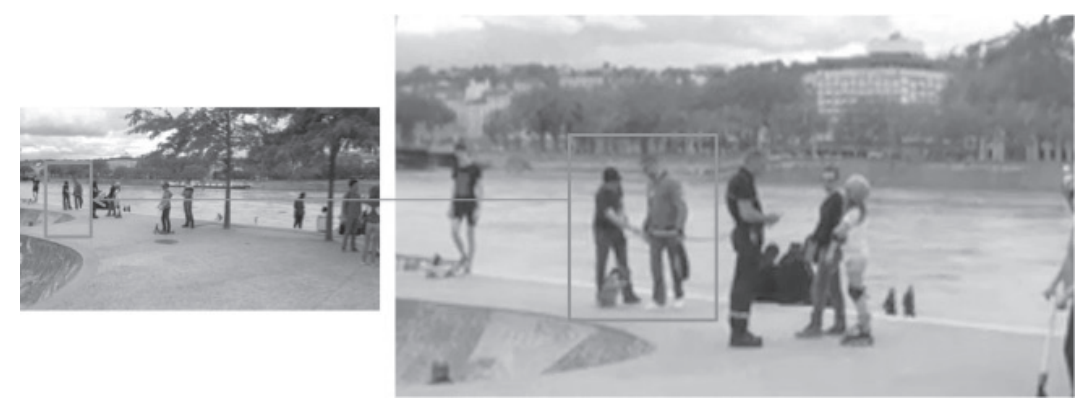

Figure 7. Dispositif d'enregistrement et préservation de la face

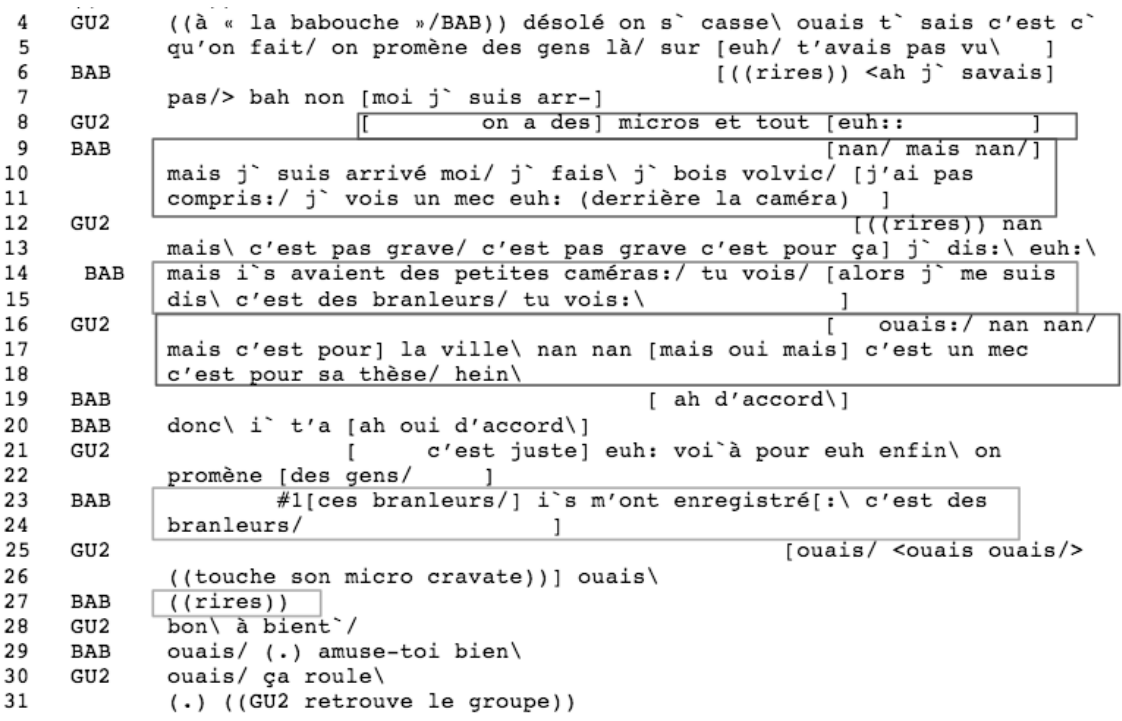

Transcription 3 - Des branleurs

Ce qui nous intéresse dans cet extrait, pour une réflexion sur l'élaboration d'une position actorielle de/par la caméra, c'est la manière dont $\mathrm{BAB}$ a perçu la présence des caméras dans l'interaction. Il ne sait pas que l'activité en cours est une balade urbaine (lignes 6-7) se déroulant dans un cadre spécifique et enregistrée (lignes 9-11) pour des finalités de recherche, comme lui explicite GU2 (lignes 16-18). Par l'explicitation de l'activité en cours et de la présence de caméras, nous verrons que cet objet se trouve être le vecteur de valorisations a priori indéterminées, non envisagées par nous-même en amont du terrain. 


\subsection{Assimilation et distanciation du chercheur au terrain}

Dans cette séquence, se déploient tout d'abord une monstration et une thématisation du dispositif par l'intervention de GU2 (ligne 8) : «on a des micros et tout ». Ensuite, BAB s'excuse pour les paroles prononcées auparavant, à côté des participants - excuse initiée aux lignes 6-7 et poursuivie aux lignes 9-11: «mais moi $\mathrm{j}$ suis arrivé moi $\mathrm{j}$ fais $\mathrm{j}$ 'bois volvic j'ai pas compris ». GU2 ratifie et valide cette excuse en rassurant BAB (ligne 12-13) : «c'est pas grave ». BAB (lignes 14-15) explicite alors la perception qu'il avait des participants manipulant les caméras en portant atteinte à leur face (Goffman, 1974) : «ìs avaient des petites caméras tu vois alors $j$ 'me suis dit c'est des branleurs ». GU2 opère alors un mouvement de réparation de la face des participants (en l'occurrence VH2 et nousmême), de crédibilisation et de légitimation de la pratique d'enregistrement (lignes 16-18) : cette balade urbaine est réalisée pour «la Ville» de Lyon et son enregistrement se déroule dans le cadre d'une thèse ${ }^{18}$. À la suite de quoi BAB rit en voyant et en touchant le micro de GU2 (lignes 23-24 et figure 7) : on pourrait inférer qu'il s'agit là d'un rire par anticipation au fait que l'on aura accès à son discours lors de son écoute, de manière ultérieure.

Cet échange est traversé par une tension entre la pratique de l'objet caméra dans un espace social particulier (scène locale) et les raisons pour lesquelles cet objet est mobilisé. Le fait que GU2 désambigüise, caractérise et légitime notre présence apporte à $\mathrm{BAB}$ un autre regard sur la scène et sur les relations actorielles en jeu. En cela même, on voit bien en quoi le fait de donner une caméra au groupe participe de l'élaboration de positions actorielles indéterminées a priori dans un espace social ouvert. À cet égard, le pluriel dans l'expression «des branleurs» est tout à fait éloquent. Si nous sommes présent à cette balade urbaine dans le cadre de notre recherche, VH2 quant à lui se fait une expérience de la balade pour des intérêts autres. Mais, comme nous manipulions tous deux une caméra, nous avons été assimilés l'un à l'autre et ramenés au même statut par BAB. Dès lors, quelles sont les précautions à prendre par rapport à un environnement de valeurs indéterminé ? En amont de l'interaction, dans quelle mesure évaluer et projeter les positions dans lesquelles on pourrait mettre les participants tout en sachant que nous ne pouvons avoir de certitude quant à la teneur et à la prise du récit dans une interaction à venir ?

\subsection{L'exploitation des ressources vidéo : quelles limites (s')imposer?}

Dans le passage ci-dessus, on voit que BAB avait une perception particulière de la présence de caméras dirigées vers les deux guides, représentants d'une culture skateboardistique. Mais que ce se passerait-il si VH2 et nous-même avions été «des branleurs », non seulement pour BAB, mais aussi pour GU2 ? Dans quelle position serions-nous vis-à-vis de GU2 ? Et, à un autre niveau, si les participants insultent ou portent atteinte à la face à d'autres acteurs de la ville (institutionnels ou non), comment remédier leur discours dans la recherche sans les compromettre ? En d'autres termes, la question se pose ici de déterminer la mesure dans laquelle un matériau peut être constitué, conservé et exploité lorsqu'il menace sa propre face ou celle d'autrui. Si la caméra constitue une ressource incontournable pour qui s'intéresse aux dimensions multimodales et praxéologiques des pratiques sémiotiques, celle-ci émerge comme un objet culturel aux valeurs indéterminées $a$ priori. Bien que cet objet puisse paraitre inerte et univoque, sa place n'est pas sans effet dans une interaction dont le périmètre est constamment renégocié : si $\mathrm{BAB}$ trouvait que

\footnotetext{
${ }^{18}$ En arrière plan, la question se pose à nous de savoir s'il n'y a pas également une réparation de la face de ceux filmés par ces caméras, en l'occurrence GU1 et GU2 : ils n'accepteraient pas de se faire filmer par des « branleurs ».
} 
VH2 et nous-mêmes étions « des branleurs », qu'en est-il de l'avis des passants et des coparticipants?

Dans l'enquête du sens des pratiques culturelles, la sémiotique ne peut ici se borner à l'analyse de pratiques déjà réalisées. Cette discipline qui étudie les médiations doit aussi porter un regard sur les pratiques en cours, potentielles et possibles des objets culturels. Dans la pratique de la recherche scientifique ou dans des pratiques sociales qui relèvent d'autres domaines, une réflexion sur l'objet caméra permet ainsi d'évaluer les limites de son propre faire dans une scène locale. À partir d'une telle évaluation, on voit se profiler le possible (et nécessaire ?) développement d'une déontologie des pratiques de la recherche en sciences du langage. Cette déontologie serait motivée par l'élaboration d'un socle commun pour une citoyenneté des pratiques de la recherche, "pour chacun", plutôt que par la restriction et l'assignation à un mode de faire unique, "pour tous".

\section{Conclusions et perspectives}

Notre proposition s'inscrit dans une réflexion qui dépasse notre objet d'étude et notre manière de faire. En vue de déployer un dialogue entre la sémiotique (Basso Fossali, 2008 ; Fontanille, 2011 ; Dondero, 2014), l'analyse conversationnelle (Mondada, 2006 ; Traverso, 2014), l'anthropologie (Lallier, 2001) et la sociologie (Pecqueux, 2012) pour une analyse située des pratiques culturelles, nous avons porté un regard sur la posture du chercheur dans un terrain spécifique. Par l'analyse d'une pratique in situ de la caméra, nous avons cherché à établir un cadre de travail à partir duquel élargir le prisme de réflexion. En effet, pour porter une attention à la (re)création d'un environnement de valeurs sémiotiques de manière stabilisée et éphémère, l'enquête sur l'élaboration des positions actorielles du chercheur et des acteurs sociaux en pratique, en interaction et en parcours, est pour nous un passage obligé.

En guise de conclusion ouverte, on propose quelques éléments sur la pratique du terrain en tant qu'expérience en hors-piste. L'enregistrement met en effet le chercheur et les participants en tension entre des inférences par analepse (on mobilise une pratique introjectée de la caméra) et des intuitions par prolepse (moments de créativité et de lâcher prise sur les données) : dans la prise de données, le chercheur ne peut développer son propre point de vue qu'en mobilisant un savoir-faire à déployer dans l'ici et maintenant, tout en ne maîtrisant pas à l'avance le paysage des pratiques auxquelles il prendra part.

Par notre proposition, la caméra n'apparaît pas comme une ressource complexe en ellemême : elle l'est par rapport aux pratiques qui en sont faites. En effet, celle-ci est complexe par l'expérience intersubjective d'un environnement qu'elle participe à la fois à stabiliser et à élaborer. Dans d'autres situations d'interactions et d'autres environnements langagiers, la caméra pourrait être plus ou moins problématique pour l'émergence et l'attestation d'un milieu spécifique: on pense notamment aux données sensibles lors des consultations médicales (projet REMILAS, en cours). L'enregistrement vidéo participe ainsi au développement d'une sémiotique vive (Basso Fossali, 2008) et au déploiement d'un regard sur les vécus de signification qui implique une analyse sémiotique in live, dans le cours de la parole en interaction, ici dans l'espace urbain.

Donner une caméra aux participants met les ressources de la recherche en tension sur une piste qui n'est pas damée et dans un champ à sillonner. D'une part, la caméra crée une perspective à laquelle nous n'aurions pas accès en mobilisant un autre dispositif (entretiens et questionnaires). D'autre part, elle rend d'autant plus complexe la documentalisation et la textualisation des pratiques interactionnelles in situ et in vivo, mais aussi les pratiques d'analyse et d'interprétation des données (durée, densité et foisonnement des échanges).

Pour le chercheur, il semble inévitable d'enquêter sur les signes qui permettent d'évaluer et de mesurer les niveaux de réflexivité et de réflexion suffisants, à toutes les étapes du travail 
de terrain. L'enjeu primordial dans la constitution de ce type de données est alors d'opérer un déplacement du regard, d'essayer de se mettre à la place de l'autre depuis son propre point de vue tout en affirmant un positionnement. Ce déplacement permettrait ainsi au chercheur de d'évaluer et d'ajuster les "bonnes mesures", à la fois spatiales et éthiques, entre distance et proximité, afin de trouver la "bonne voix" selon un faire dialogique.

\section{Bibliographie}

Astruc A. (1992). Du stylo à la caméra et de la caméra au stylo : écrits, 1942-1984, Paris : L'archipel.

Basso Fossali P. (2008). Vissuti di significazione. Temi per una semiotica viva [Vécus de signification. Thèmes pour une sémiotique vive], Pisa : ETS.

Basso Fossali P. (2012). Possibilisation, disproportion, interpénétration : trois perspectives pour enquêter sur la productivité de la notion de forme de vie en sémiotique. Actes Sémiotiques, $n^{\circ}$ 115. [URL : http://epublications.unilim.fr/revues/as/2673].

Basso Fossali P. (2015). Émancipation et disproportion : pour une problématisation de la notion de culture en sémiotique. In P. Marillaud et R. Gauthier (éds.), Cultures et valeurs: la transmission des discours, des objets et des pratiques. Actes du XXXV Colloque d'AlbiLangages et Signification, Toulouse : Université Jean-Jaurès, p. 65-81.

de Certeau M. (1980). L'invention du quotidien 1 : arts de faire, Paris : Gallimard.

Dondero M. G. (2014). Sémiotique de l'action : textualisation et notation. CASA, vol. 12, $\mathrm{n}^{\circ}$ 1, p. 15-47.

Dubois P. (1983). L'acte photographique, Paris : Nathan Université.

Eco U. (1979). Lector in fabula, Bompiani : Milano ; (1985). Lector in fabula, Le rôle du lecteur ou la Coopération interprétative dans les textes narratifs, Paris : Grasset.

Fontanille J. (2011). L'analyse du cours d'action : des pratiques et des corps, Semen, vol. 32, p. 131-158.

Geertz C. (1973). The Interpretation of Cultures, New York: Basic Books.

Gibson J. J. (1979). The Ecological Approach to Visual Perception, Boston: Houghton Mifflin.

Goffman E. (1973). La mise en scène de la vie quotidienne, Paris : Éditions de Minuit.

Greimas A. J. (1976). Sémiotique et sciences sociales, Paris : Seuil.

Greimas A. J. (1984). Sémiotique figurative et sémiotique plastique, Limoges: Presses Universitaires de Limoges.

ten Have P. (1990). Doing Conversation Analysis : A Practical Guide, London: Sage.

Kerbrat-Orecchioni C. (2005). Le discours en interaction, Paris : Armand Colin.

Labov W. (1972). Sociolinguistic Patterns, Philadelphia: University of Pennsylvania Press.

Lallier C. (2009). Pour une anthropologie filmée des interactions sociales, Paris : Éditions des Archives contemporaines.

Landowski E. (2009). Avoir prise, donner prise. Actes Sémiotiques, nº 112.

[URL : http://epublications.unilim.fr/revues/as/2852] 
Licoppe C. (2012). Les formes de la présence. Revue française des sciences de l'information et de la communication, vol. 1.

[URL : https://journals .openedition.org/rfsic/142]

Lynch M. (2002). From naturally occurring data to naturally origanized ordinary activities: Comment on Speer. Discourse Studies, vol. 4, n 4, p. 531-537.

Mondada L. (2006). Video recording as the reflexive preservation and configuration of phenomenal features for analysis. In H. Knoblauch, B. Schnettler, J. Raab \& H. G. Soeffner (Eds.), Video Analysis: Methodology and Methods. Qualitative Audiovisual Data Analysis in Sociology, Berne : Peter Lang, p. 51-68.

Mondada L. (2012). Organisation multimodale de la parole-en-interaction: pratiques incarnées d'introduction des référents. Langue française, vol. 175, n 3, p. 129-147.

Ouellet P. (2000). Poétique du regard. Littérature, perception, identité, Limoges : PULIM / Sillery : Les éditions du Septentrion.

Pecqueux A. (2012). Pour une approche écologique des expériences urbaines. Tracés, vol. 22, Lyon : ENS Éditions, p. 27-41.

Pecqueux A. (2014). Tordre l'attention. Ajustements perceptifs en situation. In Y. Citton (dir.), L'économie de l'attention. Nouvel horizon du capitalisme? Paris : La Découverte, p. 219-228.

Pike K. (1967). Language in relation to a unified theory of the structure of human behavior, The Hague: Mouton.

Projet ANR REMILAS, REfugiés, Migrants, et leurs LAngues face aux Services de santé, coordonné par Véronique Traverso, partenariat Laboratoire ICAR (UMR 5191) et Centre Orspere-Samdarra (Hôpital Le Vinatier, Lyon).

[URL : http://www.icar.cnrs.fr/sites/projet-remilas/]

Sacks H. (1984). Notes on Methodology. In J. M. Atkinson and J. Heritage (Eds.), Structures of Social Action: Studies in Conversation Analysis, Cambridge: Cambridge University Press, p. 21-27.

Schegloff E. (1998). Body Torque. Social Research, vol. 65, n 3, p. 535- 596.

Speer S. A. (2002). 'Natural' and 'contrived' data: A sustainable distinction? Discourse Studies, vol. 4, n 4, p. 511-526.

Speer S. A. \& Hutchby I. (2003). From ethics to analytics: Aspects of participants' orientations to the presence and relevance of recording device. Sociology, vol. 37, p. 315337.

Thibaud J.-P. (2002). Visions pratiques en milieu urbain. In J.-P. Thibaud, Regards en action : vers une ethnométhodologie de l'espace public, Grenoble : À la croisée, p. 21-54.

Thiburce J. (en cours). Le dialogisme urbain. De l'usage tacite des espaces publics aux formes d'appropriation narrative et affective de la ville. Thèse de doctorat sous la direction de P. Basso Fossali, Université Lumière Lyon 2, Laboratoire ICAR (UMR 5191), École doctorale 484 3LA.

Traverso V. (2014). Compétences montrées, compétences partagées, compétences situées : nomination et définition des objets dans les visites guidées. In S. Bornand (éd.), Compétences et performances. Perspectives interdisciplinaires sur une dichotomie classique, Paris : Karthala, p. 137-163. 